\title{
Magnetization transfer imaging to assess tumour response after chemoradiotherapy in rectal cancer
}

\author{
Milou H Martens ${ }^{1,2,3}$ • Doenja M. J. Lambregts ${ }^{1} \cdot$ Nickolas Papanikolaou $^{4}$ •

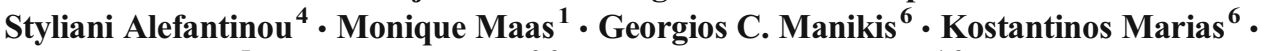 \\ Robert G. Riedl ${ }^{5}$ - Geerard L. Beets ${ }^{2,3}$ • Regina G. H. Beets-Tan ${ }^{1,3}$
}

Received: 27 December 2014 / Revised: 10 May 2015 / Accepted: 20 May 2015 / Published online: 12 June 2015

(C) The Author(s) 2015. This article is published with open access at Springerlink.com

\begin{abstract}
Purpose Single-slice magnetization transfer (MT) imaging has shown promising results for evaluating postradiation fibrosis. The study aim was to evaluate the value of multislice MT imaging to assess tumour response after chemoradiotherapy by comparing magnetization transfer ratios (MTR) with histopathological tumour regression grade (TRG).

Materials and Methods Thirty patients with locally advanced rectal cancer (cT3-4 and/or cN2) underwent routine restaging MRI 8 weeks post-chemoradiotherapy, including multislice MT-sequence, covering the entire tumour bed. Two independent readers delineated regions of interest on MTR maps, covering all potential remaining tumour and fibrotic areas. Mean MTR and histogram parameters (minimum, maximum, median, standard deviation, skewness, kurtosis, and 5-30-70-95th percentiles) were calculated. Refer-
\end{abstract}

Regina G. H. Beets-Tan

beetstan@me.com

1 Department of Radiology, Maastricht University Medical Center, PO Box 5800, 6202 AZ Maastricht, The Netherlands

2 Department of Surgery, Maastricht University Medical Center, Maastricht, The Netherlands

3 GROW School of Oncology and Developmental Biology, Maastricht University Medical Center, Maastricht, The Netherlands

4 N. Papanikolaou and Associates LLC, Heraklion, Greece

5 Department of Pathology, Maastricht University Medical Center, Maastricht, The Netherlands

6 Computational Medicine Laboratory, Institute of Computer Science, Foundation for Research and Technology, Hellas, Greece ence standard was histological TRG1-2 (good response) and TRG3-5 (poor response).

Results 24/30 patients were male; mean age was $67.7 \pm$ 10.8 years. Mean MTR rendered AUCs of 0.65 (reader1) and 0.87 (reader2) to differentiate between TRG1-2 versus TRG3-5. Best results were obtained for $95^{\text {th }}$ percentile (AUC 0.75- 0.88). Interobserver agreement was moderate (ICC 0.50) for mean MTR and good (ICC 0.80 ) for $95^{\text {th }}$ percentile.

Conclusions MT imaging is a promising tool to assess tumour response post-chemoradiotherapy in rectal cancer. Particularly, $95^{\text {th }}$ percentile results in AUCs up to 0.88 to discriminate a good tumour response.

Key Points

- The mean MTR can differentiate between good and poor responders after chemoradiation.

- In addition to measurement of the mean value, histogram analyses can be beneficial.

- The histogram parameter $95^{\text {th }}$ percentile can reach AUCs of $0.75-0.88$.

Keywords Magnetization transfer imaging · Rectal cancer . Response assessment . Tumour regression grade .

Interobserver agreement

\section{Introduction}

The assessment of tumour response after chemoradiotherapy (CRT) in patients with rectal cancer is becoming increasingly relevant. Until recently, chemoradiotherapy followed by surgical resection of the tumour was the standard, irrespective of the response to treatment [1]. However, organ-preserving 
treatments in carefully selected patients showing a clinically complete or near-complete response are now considered potential-albeit still controversial-alternatives to resection. Alternative options include local excision for a small tumour remnant [2] and non-operative management ("watch-andwait" policy) in the case of a complete tumour response [3-5]. Critical in these organ-preserving treatments is the availability of tools to accurately select suitable patients. In studies reported to date so far, this has been mainly done by clinical examination and endoscopy [6]. A restaging MRI 8 weeks after chemoradiotherapy has become standard in many centres, as MRI has proven valuable in assessing tumour downsizing, identifying tumour regression from initially invaded structures, and thereby altering the surgical approach [7]. However, one key challenge in MRI remains the interpretation of post-radiation fibrosis [8]. With morphological MRI, it is very difficult to discriminate between fibrosis with and without tumour remnants [9], as was illustrated in a recent meta-analysis that reported a poor sensitivity of $19 \%$ for MRI in discriminating complete response (pCR) from residual tumour [10]. Quantification of signal intensities on T2weighted MRI has been suggested as helpful: a greater reduction in signal intensity after chemoradiation is observed in complete responders, with a more homogeneous signal distribution compared to patients with residual tumours. Thus far, this approach has been addressed by only a single report [11]. Various studies have reported promising results with functional imaging techniques such as diffusion-weighted imaging and dynamic contrast-enhanced MRI for the detection of residual tumour after CRT [12-14], but these techniques do not specifically target fibrosis.

Recent reports have shown that "magnetization transfer" (MT) imaging may also be a helpful tool for assessing bowel fibrosis [15-17]. MT imaging is a technique that utilizes differences in the magnetization interaction of free 'unbound' water protons and macromolecular-bound protons. After an MT pulse is applied, there is a transfer of magnetization from free water protons towards macromolecular-bound water protons, which can only occur when macromolecular-bound water protons are present within a given tissue. In tissues that are rich in macromolecules (which is typically the case in fibrosis due to the presence of collagen), the transfer of magnetization-expressed as the "magnetization transfer ratio" or MTR - will be high. In a recent proof-of-principle study of 26 patients with rectal cancer who were treated with preoperative CRT, the MTR of histopathologically proven areas of fibrosis was significantly higher than that of areas of residual tumour, indicating a potential benefit in adding MT imaging to a rectal cancer imaging protocol after CRT [16]. In this report, only a single slice of the MT sequence was acquired, which did not cover (and is therefore not representative of) the entire tumour area. As such, it is difficult to assess the value of MT imaging for evaluating the overall tumour response. Moreover, only mean MTR values were assessed, which provide a rough estimation of the MTR of a given area of interest, but do not take into account the heterogeneity of the tumour and fibrosis.

The aim of the current study, therefore, was to evaluate the potential value of multislice MT imaging of the whole tumour volume for assessing tumour response after CRT by comparing the magnetization transfer ratios with the tumour regression grade (TRG) as assessed at histopathology. A second goal was to assess the added benefit of performing histogram analyses as compared to evaluation of mean MTR values alone.

\section{Materials and Methods}

\section{Patients}

This study was performed retrospectively. According to national law, institutional review board approval was not required. All patients were informed that their anonymized data could be used for research purposes, to which none of the study patients objected. Between November 2012 and June 2014, all consecutive patients with locally advanced rectal cancer were considered for inclusion. Inclusion criteria were (1) biopsy-proven rectal adenocarcinoma, (2) locally advanced disease (cT3/4 and/or N2) without distant metastasis, (3) neoadjuvant chemoradiation, (4) availability of a restaging MRI including MT sequence, (5) resection within 2 months after restaging MRI or, in the case of non-operative treatment and follow-up in patients with a clinical complete response, a disease-free follow-up period of $>1$ year.

\section{MR Imaging}

MR imaging was performed at $1.5 \mathrm{~T}$ (Ingenia, Philips Medical Systems, Best, the Netherlands) using a phased array body coil. Patients had received no bowel preparation, and were placed in feet-first supine position. To minimize peristaltic movement, patients received an intravenous bolus injection of $20 \mathrm{mg}$ of butylscopolamine (Buscopan; Boehringer Ingelheim BV, Ingelheim, Germany). The routine clinical MR protocol included standard T2-weighted (T2W) fast spin-echo sequences in three planes (sagittal, axial, and coronal). Additionally, an axial MTR sequence was performed, with the following parameters: multislice fast field echo, dynamic (simultaneous acquisition of images with and without MT pulse in one sequence), interleaved two-dimensional, TR/TE 50/4.6, $12^{\circ}$ flip angle, 2 NSA, $1.17 \times 1.47 \times 3.00 \mathrm{~mm}$ voxel size, 10 slices, $300 \times 239 \mathrm{~mm}$ field of view, $256 \times 162$ matrix, receiver bandwidth $147 \mathrm{~Hz} /$ pixel, acquisition time 5:02 min. The MT pulse was an on-resonance composite pulse and consisted of four 
rectangular pulses $\left(90^{\circ}-\mathrm{x} 90^{\circ} \times 90^{\circ} \mathrm{x} 90^{\circ}\right.$-) with a duration of $2 \mathrm{~ms}\left(0.5 \mathrm{~ms}\right.$ for each $90^{\circ}$ pulse $)$ and a bandwidth of $2083 \mathrm{~Hz}$. The delay between the MT pulse and the following excitation was $3.2 \mathrm{~ms}$. The axial T2-weighted and MT sequences were angulated in identical planes perpendicular to the (former) tumour axis as defined on sagittal MRI.

\section{Image evaluation and calculation of magnetization transfer ratio (MTR) parameters}

Image assessment was performed on the restaging MRI routinely performed 8 weeks after completion of CRT. Magnetization transfer ratio maps were generated using a freely distributed software tool (MANGO [Multi-image Analysis GUI], version 2.6; Research Imaging Institute, University of Texas Health Science Center, San Antonio, TX, USA). MTR maps were generated using the following formula on a voxel-byvoxel basis: MTR $=100 \times\left(S I_{\text {off }}-S I_{\text {on }}\right) / S I_{\text {off }}$, where $S I_{\text {off }}$ and $S I_{o n}$ represent the signal intensity with the MT pulse turned off and on, respectively. Two independent readers with 6 (DMJL) and 3 years (MHM) of specific expertise in rectal cancer imaging manually delineated free-hand regions of interest (ROIs) on the MTR maps on each individual tumourcontaining slice. The corresponding T2-weighted images as well as the primary staging MRIs were at the readers' disposal as an anatomical reference and for determining the primary tumour location (Fig. 1). On every slice, the entire area of potential residual tumour was included within the ROI, including any potential viable tumour remnants (isointense mass or wall thickening) and fibrosis (hypointense wall thickening). The two readers were blinded to each other's results and histopathology outcomes. Histogram analyses were performed using in-house software, developed in the programming environment R language [18]. The MTR measurements were imported, and the following normalized histogram parameters were calculated automatically: minimum, maximum, median, standard deviation, skewness (asymmetry of distribution), kurtosis (width of peak of distribution), and 5th, 30th, 70th, and 95 th percentiles.

\section{Standard of reference}

Histopathological assessment of the surgical resection specimens served as the primary standard of reference. This was performed according to the method described by Quirke et al. [19]. A dedicated GI pathologist evaluated the tumour regression grade (TRG) according to the method reported by Mandard [20]: TRG 1 (complete regression) $=$ absence of residual cancer and fibrosis extending to the different layers of the bowel wall; TRG 2 = rare residual cancer cells scattered throughout predominantly fibrosis; TRG $3=$ increase in the number of residual cancer cells but fibrosis still predominates; TRG 4 = residual cancer outgrowing fibrosis; and TRG $5=$
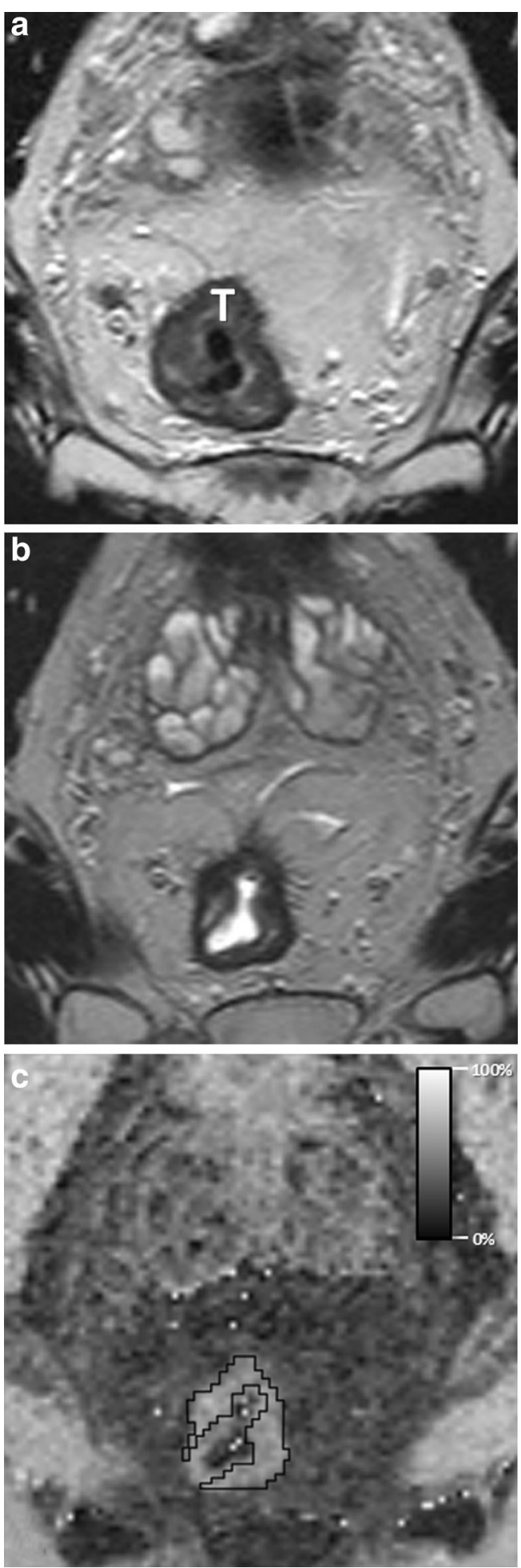

Fig. 1 a Axial T2-weighted MRI at primary staging, with ' $T$ ' indicating the primary tumour. b Axial restaging T2-weighted MRI performed 8 weeks after chemoradiation, and $\mathbf{c}$ corresponding axial MTR map showing delineation of the tumour remnant

absence of any regressive changes. Patients were then dichotomized into two categories: TRG 1-2 (good response) and TRG 3-5 (poor response). 


\section{Statistical analyses}

Statistical analyses were performed with SPSS statistical software version 20.0 (IBM Corp., Armonk, NY, USA). Intraclass correlation coefficients (ICCs) and Bland-Altman plots were calculated to determine interobserver agreement for the MTR measurements between the two readers. Receiver operator characteristic (ROC) curves were computed, and corresponding areas under the curve (AUCs) with $95 \%$ confidence intervals were calculated to assess the diagnostic performance of the various MTR histogram parameters for discriminating between TRG1-2 and TRG3-5. The predictive value of parameters was assessed by logistic regression analyses; parameters were selected for multivariable analyses when $p$ was $<0.20$. For the predicted probabilities for the combined MTR histogram parameters, AUCs with $95 \%$ confidence intervals were calculated.

\section{Results}

\section{Patient and treatment characteristics}

Forty-one consecutive patients were considered for inclusion. Eleven patients were excluded for the following reasons: delayed surgery after CRT $(n=5)$, mucinous tumour $(n=4)$, and refusal of surgery despite clinical residual tumour $(n=2)$. The inclusion process and reasons for exclusion are displayed in Fig. 2. In total, 30 patients were included ( 24 male, 6 female; mean age $67.7 \pm 10.8$ years). Preoperative treatment consisted of a long course of chemoradiation $(28 \times 1.8 \mathrm{~Gy}+2 \times 825 \mathrm{mg} /$ $\mathrm{m}^{2} /$ day capecitabine) in 28 patients. The remaining two patients received $5 \times 5$ Gy, with an interval of $8-10$ weeks before surgery. The median interval between the final day of radiation and restaging MRI was 8.9 weeks (range 6.6-24.7). Eighteen patients underwent a low anterior resection, two underwent an abdominoperineal resection, and two underwent transanal endoscopic microsurgery. Histological tumour regression grades in these patients were as follows: TRG $1(n=4)$, TRG $2(n=5)$,
TRG $3(n=7)$, TRG $4(n=5)$, and TRG $5(n=1)$. Eight patients were followed for $>1$ year according to a watch-and-wait policy (median disease-free survival of 17.5 months, range 12-21 months). These patients were all considered TRG1. In total, the dichotomized tumour regression grade was as follows: $n=17$ TRG1-2 and $n=13$ TRG3 -5 .

\section{Interobserver agreement}

The intraclass correlation coefficients (ICCs) between the two readers for the mean MTR and various histogram parameters are given in Table 1. The ICC for the mean MTR measurements was 0.50 (moderate). The highest ICCs were obtained for the maximum MTR and the 95th percentile, with an ICC of 0.80 (good). Figure 3 displays the Bland-Altman plot for the mean MTR measurements and for the 95 th percentile. The mean bias in mean MTR between the two readers was -1.79 , and limits of agreement were \pm 5.70 . For the 95 th percentile, the mean bias was -1.22 and limits of agreement were \pm 4.67 .

\section{MTR and histogram parameters}

The mean MTR was $59.6 \pm 3.4$ (TRG1-2) versus $58.4 \pm 3.1$ (TRG3-5) for reader $1(p=0.33)$ and $62.1 \pm 1.9($ TRG1-2) versus $59.2 \pm 1.9$ (TRG3-5) for reader $2(p<0.001)$. Figure 4 shows the distribution in mean MTR values (averaged for the two readers) among the tumour regression grades. The mean 95th percentile was $73.9 \pm 4.9$ (TRG1-2) versus $70.9 \pm 1.8$ (TRG3-5) for reader $1(p=0.029)$ and $75.7 \pm 3.0$ (TRG1-2) versus $71.4 \pm 2.4$ (TRG3-5) for reader $2(p<0.001)$. Table 2 shows the results of the receiver operator characteristic curve analyses for discriminating between TRG 1-2 and TRG 3-5 for the various histogram parameters as well as for the combined assessment of (1) mean MTR + skewness (2) mean MTR +95 th percentile, (3) skewness +95 th percentile, and (4) mean MTR + skewness +95 th percentile. The latter combinations were derived from the logistic regression analyses. The best single parameter for discriminating between TRG 1-2 and TRG 3-5 was the 95th percentile for both readers (AUC 0.75
Fig. 2 CONSORT diagram of patient recruitment and reasons for exclusion

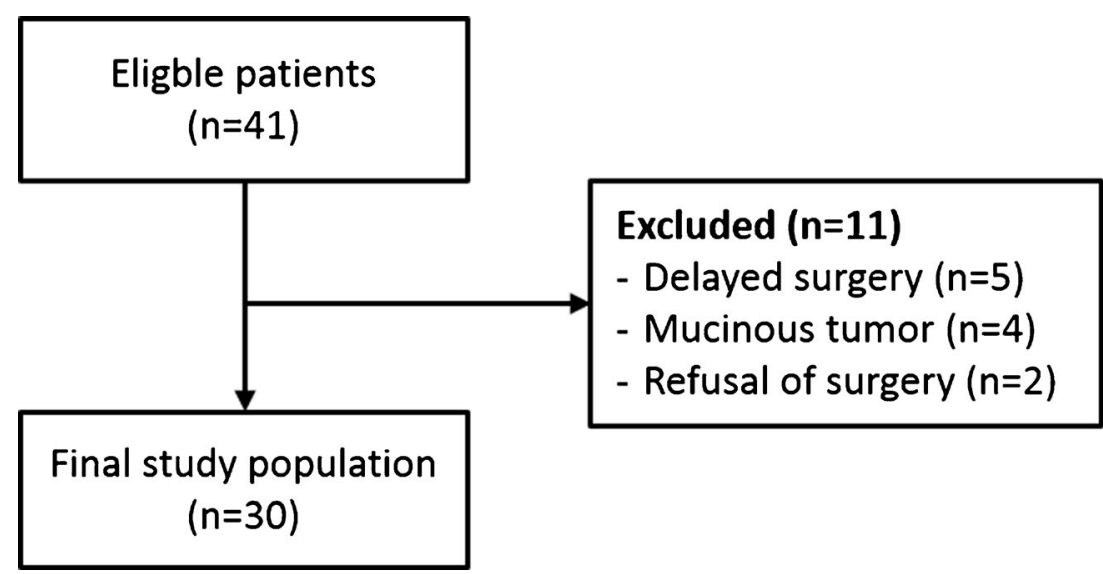



correlation coefficients for the mean MTR and the histogram parameters measured after chemoradiation
Table 1 Intraclass

\begin{tabular}{ll}
\hline & Intraclass correlation \\
\hline $\begin{array}{l}\text { Mean MTR } \\
\text { Mean }\end{array}$ & 0.50 \\
Histogram parameters \\
Min & 0.12 \\
Max & 0.80 \\
Median & 0.55 \\
SD & 0.53 \\
Skewness & 0.29 \\
Kurtosis & 0.36 \\
5th percentile & 0.29 \\
30th percentile & 0.45 \\
70th percentile & 0.64 \\
95th percentile & 0.80 \\
\hline
\end{tabular}

for reader 1 and 0.88 for reader 2). The various combinations of mean MTR, skewness, and 95th percentile resulted in AUCs of $0.72-0.81$ for reader 1 and $0.87-0.90$ for reader 2 . This was not a significant improvement compared with the 95 th percentile as a single parameter $(p=0.28-1.00)$. Measurements of sensitivity, specificity, positive predictive value, negative predictive value, and overall accuracy of the 95th percentile and the various combinations are presented in Table 3. Sensitivity and specificity for diagnosing a good response were in a range of $71-77 \%$ and $77-92 \%$, respectively.

\section{Discussion}

The purpose of this study was to evaluate the potential value of whole-tumour MT imaging to assess tumour response after neoadjuvant chemoradiation for rectal cancer. We compared the mean MTR and various histogram parameters derived from the MTR between patients with a good response after CRT (TRG 1-2) and those with a poor response (TRG 3-5). Results showed that MT imaging of the whole tumour volume after CRT could predict a TRG1-2 at histology with AUCs up to 0.88 (for the 95 th percentile), thereby confirming that MTR may be useful for assessing fibrosis.

Interestingly, the best results were obtained for the histogram parameters, and in particular for the 95th percentile, which resulted in high AUCs for both readers 1 and $2(0.75$ and 0.88 , respectively). The 95 th percentile represents the value above which the highest $5 \%$ of the MTR measurements are within a given ROI. In other words, the 95th percentile represents the most fibrotic areas within the ROI. It is anticipated that the 95th percentile will be higher in patients with a good response (with predominant fibrosis) and lower in patients with a poor response. This is indeed supported by the findings of the current study. It also confirms our secondary
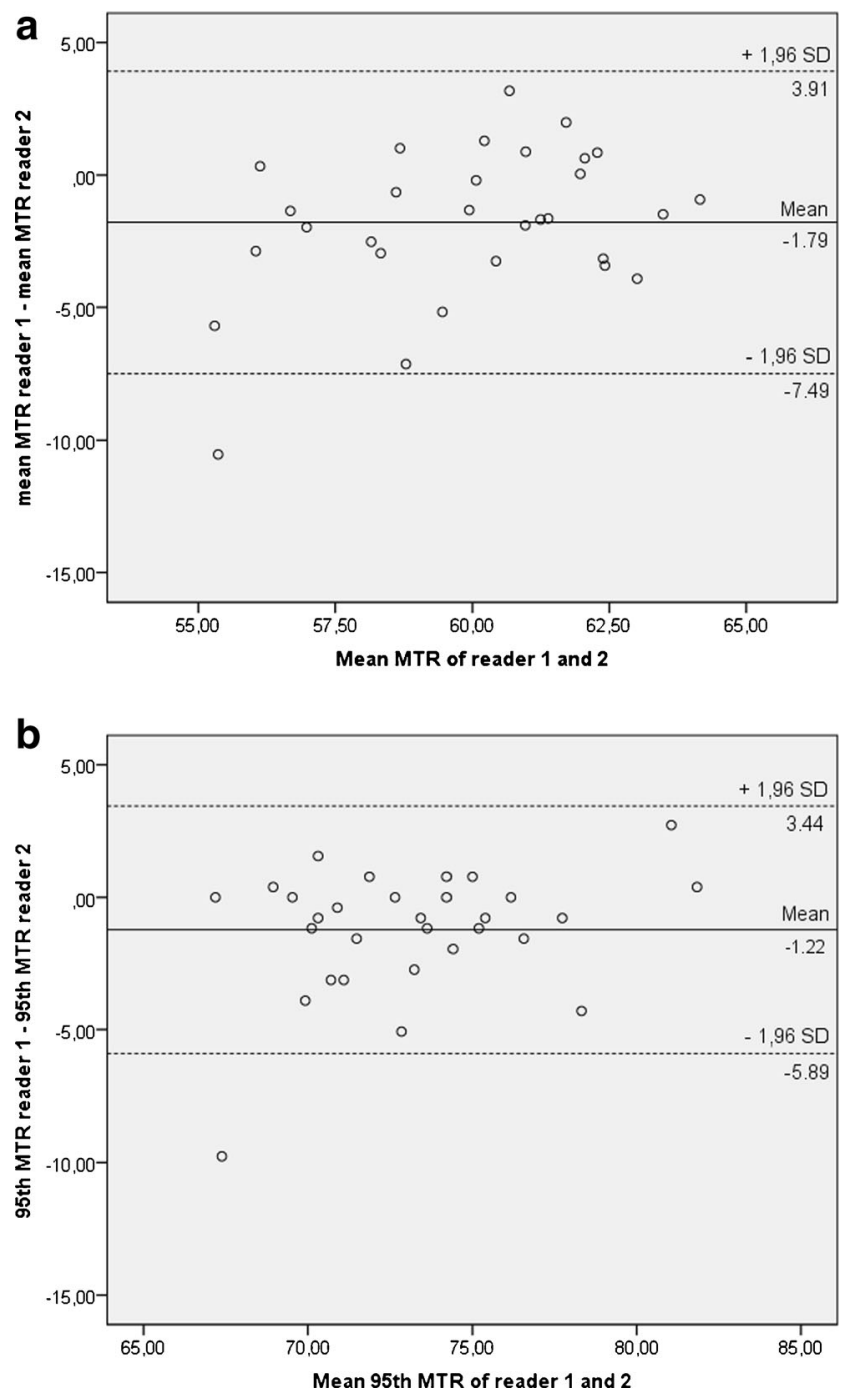

Fig. 3 Interobserver reproducibility for mean MTR measurements (a) and 95th percentile MTR (b). Bland-Altman plot of the averaged measurement of the two readers (x-axis) plotted against the difference in measurement between the two readers (y-axis). The continuous line represents the mean absolute difference (bias) between the two readers; the dashed lines represent the $95 \%$ confidence intervals of the mean difference (limits of agreement)

hypothesis that, compared to analysis of only mean MTR, histogram data analysis is advantageous in that it gives us better insight into the distribution pattern of MTR and therefore of the heterogeneity of the tumour area. Moreover, with a good intraclass correlation coefficient of 0.80 , the 95 th percentile appears to be more robust than mean MTR values, which showed only a moderate ICC of 0.50 . Interobserver reproducibility may thus be a limiting factor when assessing MTR. For the mean MTR measurements, the difference observed between patients with TRG1-2 and TRG3-5 was, in fact, smaller than the limits of agreement for the difference in MTR measurements between the two readers as derived from the Bland-Altman analysis. Although ICC was considerably better for the 95th percentile, the mean difference between the 
Fig. 4 Boxplot showing the distribution of mean MTR values between the different TRG subgroups. MTR values were averaged between the two readers

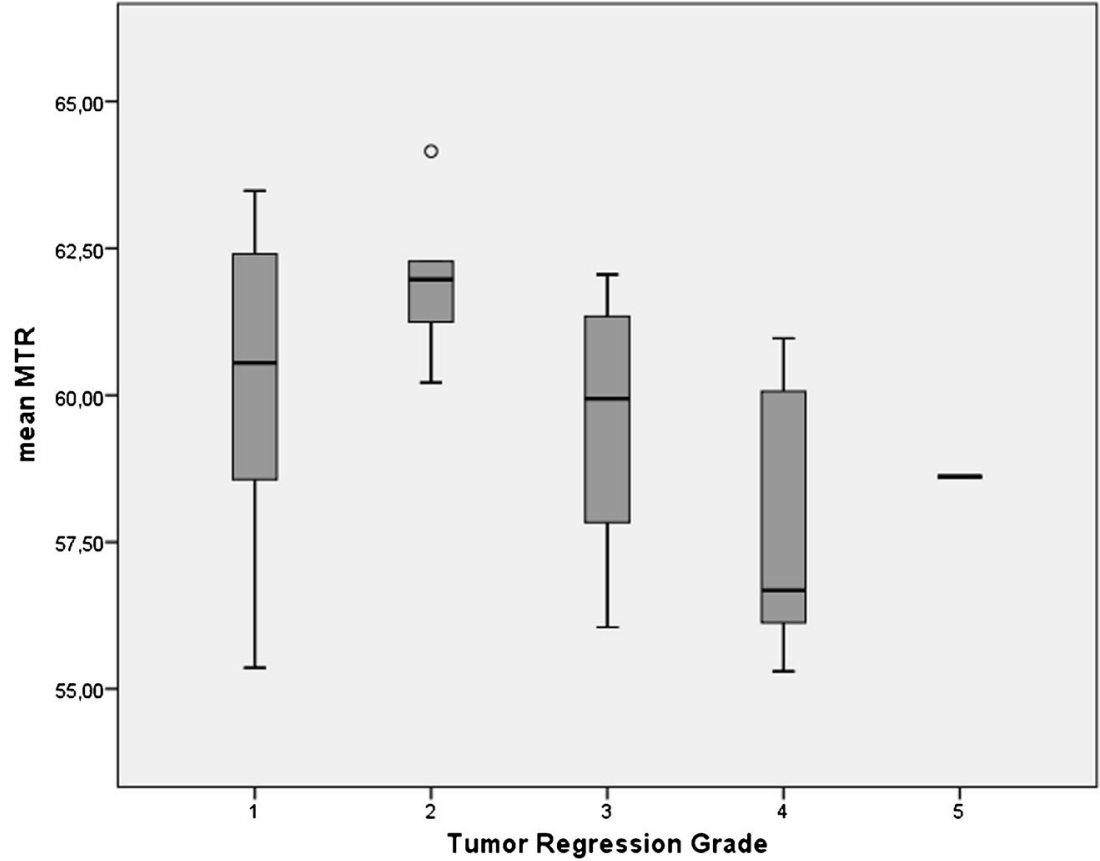

two response groups was still smaller than the differences in measurements between the two readers. While this is limiting with regard to clinical utility, it may be partially explained by the current study methodology, in which ROI placement was performed directly on the MTR maps (see Fig. 1), which lack anatomical detail, making them challenging for radiologists to interpret. Although corresponding T2-weighted images were used for anatomical reference during the delineation process, it may be worthwhile investigating whether it would be helpful to draw the ROIs and copy them from another sequence, such as the standard T2-weighted images. A second disadvantage of quantitative imaging such as MT imaging is that it can be a somewhat incomprehensible concept for clinicians to interpret. A simpler, qualitative approach such as an MRIbased scoring system may be easier for clinicians to understand. The Royal Marsden research group have investigated
Table 2 Results from the receiver operator characteristic curve analyses for histogram parameters individually and in various combinations for in differentiating TRG1-2 from TRG3-5

\begin{tabular}{|c|c|c|c|c|}
\hline & \multicolumn{2}{|c|}{ Reader 1} & \multicolumn{2}{|c|}{ Reader 2} \\
\hline & AUC & $95 \% \mathrm{CI}$ & AUC & $95 \% \mathrm{CI}$ \\
\hline \multicolumn{5}{|l|}{ Mean MTR } \\
\hline Mean & 0.65 & $0.45-0.85$ & 0.87 & $0.75-1.00$ \\
\hline \multicolumn{5}{|l|}{ Histogram parameters } \\
\hline Min & 0.67 & $0.47-0.87$ & 0.72 & $0.53-0.91$ \\
\hline Max & 0.67 & $0.45-0.88$ & 0.58 & $0.37-0.80$ \\
\hline Median & 0.62 & $0.41-0.83$ & 0.86 & $0.72-0.99$ \\
\hline $\mathrm{SD}$ & 0.62 & $0.41-0.82$ & 0.66 & $0.46-0.85$ \\
\hline Skewness & 0.72 & $0.54-0.91$ & 0.58 & $0.37-0.78$ \\
\hline Kurtosis & 0.70 & $0.51-0.89$ & 0.65 & $0.44-0.86$ \\
\hline 5th percentile & 0.50 & $0.28-0.72$ & 0.65 & $0.45-0.85$ \\
\hline 30th percentile & 0.55 & $0.34-0.77$ & 0.81 & $0.66-0.97$ \\
\hline 70th percentile & 0.68 & $0.48-0.87$ & 0.88 & $0.76-1.00$ \\
\hline 95th percentile & 0.75 & $0.56-0.94$ & 0.88 & $0.76-1.00$ \\
\hline \multicolumn{5}{|l|}{ Combined parameters } \\
\hline Mean + skewness & 0.72 & $0.54-0.91$ & 0.87 & $0.75-1.00$ \\
\hline Mean +95 th percentile & 0.75 & $0.56-0.94$ & 0.90 & $0.78-1.00$ \\
\hline Skewness +95 th percentile & 0.81 & $0.65-0.97$ & 0.89 & $0.77-1.00$ \\
\hline Mean + skewness +95 th percentile & 0.79 & $0.63-0.95$ & 0.90 & $0.78-1.00$ \\
\hline
\end{tabular}


Table 3 Diagnostic accuracy of MTR parameters

\begin{tabular}{|c|c|c|c|c|c|c|c|c|}
\hline Parameter & Reader & AUC & Cut-off & Sensitivity & Specificity & PPV & NPV & Overall accuracy \\
\hline \multirow[t]{2}{*}{ 95th percentile } & Reader 1 & 0.75 & $72.9 \%$ & 70.6 & 84.6 & 68.8 & 85.1 & 76.7 \\
\hline & Reader 2 & 0.88 & $74.0 \%$ & 70.6 & 84.6 & 68.8 & 85.7 & 76.7 \\
\hline \multirow[t]{2}{*}{ Mean +95 th percentile } & Reader 1 & 0.75 & -0.14 & 70.6 & 92.3 & 70.6 & 92.3 & 80.0 \\
\hline & Reader 2 & 0.90 & -0.06 & 76.5 & 84.6 & 73.3 & 86.7 & 80.0 \\
\hline \multirow[t]{2}{*}{ Skewness +95 th percentile } & Reader 1 & 0.81 & -0.20 & 76.5 & 84.6 & 73.3 & 86.7 & 80.0 \\
\hline & Reader 2 & 0.89 & -0.06 & 76.5 & 84.6 & 73.3 & 86.7 & 80.0 \\
\hline \multirow[t]{2}{*}{ Mean + skewness +95 th percentile } & Reader 1 & 0.79 & -0.19 & 70.6 & 76.9 & 66.7 & 80.0 & 73.3 \\
\hline & Reader 2 & 0.90 & -0.06 & 76.5 & 84.6 & 73.3 & 86.7 & 80.0 \\
\hline
\end{tabular}

$A U C=$ area under the receiver operating characteristics curve, $P P V=$ positive predicting value, $N P V=$ negative predicting value.

the mrTRG, an MRI-based scoring system of tumour regression grade, which is similar in construction to the pathological Mandard model [21]. In their report, the degree of fibrosis determined at pathology was significantly associated with the mrTRG, albeit with moderate interobserver agreement (0.55) [22]. To date, this qualitative approach has not been reproduced or validated by other groups. For future studies, it would be interesting to compare this qualitative approach with MT imaging for the assessment of fibrosis.

The results of the current study are in line with a previous proof-of-principle study in 26 patients in which a single "sample" slice of the MT sequence was obtained after CRT. In that study, the single-slice MTR map was correlated with a corresponding whole-mount histopathology section, and individual areas of fibrosis, tumour, and the normal rectal wall were compared in detail with the underlying histology. This resulted in a significant difference in mean MTR between areas of pathologically proven fibrosis and non-fibrotic areas, including residual tumour [16]. We did not perform such a detailed whole-mount correlation with histopathology, but evaluated the overall MTR of the entire tumour area (obtained from multislice MT imaging) to discriminate between good (TRG1-2) and poor responses (TRG3-5) to assess the value of measuring MTR from a more clinical, patient-based perspective. Our results confirmed that whole-tumour MTR was helpful for differentiating between response groups (i.e. patients with varying degrees of fibrosis). Nevertheless, MTR measurements mainly reflect an estimation of the amount of fibrosis, and specific information regarding the presence and extent of residual tumour cells is limited. In this respect, it might be interesting to combine MT imaging with other tools such as diffusion-weighted MR imaging that specifically target detection of residual tumour in order to obtain a more complete understanding of the overall tumour response. Diffusion-weighted imaging has shown great promise in various studies as a sensitive technique for detecting residual tumour within post-radiation fibrosis, with AUCs in the range of $0.87-$ $0.93[13,14,23,24]$. As such, the two techniques may be of complementary value in assessing the effects of chemoradiotherapy and evaluating the various aspects of treatment response.
The MT ratios in our study are higher than typically reported in the literature. This is likely explained by a combination of factors. First, we used on on-resonance MT pre-pulse, since an on-resonance pulse has been suggested to result in a more prominent MT effect (resulting in higher MT ratios) than that of an off-resonance MT pulse [25, 26], which is more commonly reported in the current literature. Other factors that may have contributed to the relatively high MT ratios in our study include the use of a multislice sequence and the dynamic acquisition of the MT sequence (simultaneous acquisition of images with and without MT pulse in one sequence). The fact that variables such as acquisition parameters can affect the MTR reveals the need for standardization and calibration of the MT-sequence.

There are some limitations to our study design. First, the patient group was relatively small. Second, there were some small differences within the study group in the neoadjuvant treatment scheme. And finally, histopathological confirmation was not obtained in all patients. Eight patients were followed according to a watch-and-wait protocol, with a recurrence-free follow-up period of 12-21 months, which was considered a surrogate endpoint for a complete response (TRG 1).

In conclusion, MT imaging is a promising tool for assessing tumour response after neoadjuvant chemoradiation in rectal cancer. In addition to measurement of mean MTR, histogram data assessment (in particular the 95th percentile) proved beneficial and resulted in high AUCs for differentiating between patients with a good and poor response. A potential limitation of MTR is the limited interobserver agreement, which may be improved by adjusting the measurement protocol, an item that should be addressed by future studies.

Acknowledgements The scientific guarantor of this publication is RGHBT. The authors of this manuscript declare no relationships with any companies whose products or services may be related to the subject matter of the article. The authors state that this work has not received any funding. One of the authors has significant statistical expertise. Institutional Review Board approval and written informed consent were not required because of the retrospective nature of this study. . Methodology: retrospective, diagnostic study, performed at one institution. The authors would like to thank Mr. Jan Verwoerd (Clinical Scientist MRI; 
Philips Healthcare Benelux) for his assistance in evaluating the MT sequence used in this study.

Open Access This article is distributed under the terms of the Creative Commons Attribution-NonCommercial 4.0 International License (http:// creativecommons.org/licenses/by-nc/4.0/), which permits any noncommercial use, distribution, and reproduction in any medium, provided you give appropriate credit to the original author(s) and the source, provide a link to the Creative Commons license, and indicate if changes were made.

\section{References}

1. Sauer R, Becker H, Hohenberger W et al (2004) Preoperative versus postoperative chemoradiotherapy for rectal cancer. N Engl J Med 351:1731-1740

2. Coco C, Rizzo G, Mattana C et al (2013) Transanal endoscopic microsurgery after neoadjuvant radiochemotherapy for locally advanced extraperitoneal rectal cancer: short-term morbidity and functional outcome. Surg Endosc 27:2860-2867

3. Habr-Gama A, Perez RO, Nadalin W et al (2004) Operative versus nonoperative treatment for stage 0 distal rectal cancer following chemoradiation therapy: long-term results. Ann Surg 240:711717, discussion 717-8

4. Maas M, Beets-Tan RG, Lambregts DM et al (2011) Wait-and-see policy for clinical complete responders after chemoradiation for rectal cancer. J Clin Oncol 29:4633-4640

5. Smith JD, Ruby JA, Goodman KA et al (2012) Nonoperative management of rectal cancer with complete clinical response after neoadjuvant therapy. Ann Surg 256:965-972

6. Habr-Gama A, Perez RO, Wynn G, Marks J, Kessler H, GamaRodrigues J (2010) Complete clinical response after neoadjuvant chemoradiation therapy for distal rectal cancer: characterization of clinical and endoscopic findings for standardization. Dis Colon Rectum 53:1692-1698

7. Vliegen RF, Beets GL, Lammering G et al (2008) Mesorectal fascia invasion after neoadjuvant chemotherapy and radiation therapy for locally advanced rectal cancer: accuracy of MR imaging for prediction. Radiology 246:454-462

8. Beets-Tan RG, Lambregts DM, Maas M et al (2013) Magnetic resonance imaging for the clinical management of rectal cancer patients: recommendations from the 2012 European Society of Gastrointestinal and Abdominal Radiology (ESGAR) consensus meeting. Eur Radiol 23:2522-2531

9. Barbaro B, Vitale R, Leccisotti L et al (2010) Restaging locally advanced rectal cancer with MR imaging after chemoradiation therapy. Radiographics 30:699-716

10. van der Paardt MP, Zagers MB, Beets-Tan RG, Stoker J, Bipat S (2013) Patients who undergo preoperative chemoradiotherapy for locally advanced rectal cancer restaged by using diagnostic $\mathrm{mr}$ imaging: a systematic review and meta-analysis. Radiology

11. Kluza E, Rozeboom ED, Maas M et al (2013) T2 weighted signal intensity evolution may predict pathological complete response after treatment for rectal cancer. Eur Radiol 23:253-261

12. Gollub MJ, Gultekin DH, Akin O et al (2011) Dynamic contrast enhanced-MRI for the detection of pathological complete response to neoadjuvant chemotherapy for locally advanced rectal cancer. Eur Radiol 22:821-831

13. Lambregts DM, Rao SX, Sassen S, et al (2014) MRI and diffusionweighted MRI volumetry for identification of complete tumor responders after preoperative chemoradiotherapy in patients with rectal cancer: a bi-institutional validation study. Ann Surg

14. Kim SH, Lee JM, Hong SH et al (2009) Locally advanced rectal cancer: added value of diffusion-weighted MR imaging in the evaluation of tumor response to neoadjuvant chemo- and radiation therapy. Radiology 253:116-125

15. Adler J, Swanson SD, Schmiedlin-Ren P et al (2011) Magnetization transfer helps detect intestinal fibrosis in an animal model of Crohn disease. Radiology 259:127-135

16. Martens MH, Lambregts DM, Papanikolaou N et al (2014) Magnetization transfer ratio: a potential biomarker for the assessment of postradiation fibrosis in patients with rectal cancer. Investig Radiol 49:29-34

17. Heijnen LA, Lambregts DM, Mondal D et al (2013) Diffusionweighted MR imaging in primary rectal cancer staging demonstrates but does not characterise lymph nodes. Eur Radiol 23: 3354-3360

18. R Development Core Team (2014) R: a language and environment for statistical computing. R Foundation for Statistical Computing, Vienna, Austria. http://www.R-project.org; Available from: http:// www.R-project.org

19. Quirke P, Durdey P, Dixon MF, Williams NS (1986) Local recurrence of rectal adenocarcinoma due to inadequate surgical resection. Histopathological study of lateral tumour spread and surgical excision. Lancet 2:996-999

20. Mandard AM, Dalibard F, Mandard JC et al (1994) Pathologic assessment of tumor regression after preoperative chemoradiotherapy of esophageal carcinoma. Clinicopathologic correlations. Cancer 73:2680-2686

21. PatelUB, Taylor F, Blomqvist L, et al Magnetic resonance imagingdetected tumor response for locally advanced rectal cancer predicts survival outcomes: MERCURY experience. J Clin Oncol 29:3753-60

22. Patel UB, Brown G, Rutten H et al (2012) Comparison of magnetic resonance imaging and histopathological response to chemoradiotherapy in locally advanced rectal cancer. Ann Surg Oncol 19: 2842-2852

23. Curvo-Semedo L, Lambregts DM, Maas M et al (2011) Rectal cancer: assessment of complete response to preoperative combined radiation therapy with chemotherapy-conventional MR volumetry versus diffusion-weighted MR imaging. Radiology 260:734-743

24. Ha HI, Kim AY, Yu CS, Park SH, Ha HK (2013) Locally advanced rectal cancer: diffusion-weighted MR tumour volumetry and the apparent diffusion coefficient for evaluating complete remission after preoperative chemoradiation therapy. Eur Radiol 23:3345-3353

25. Phinikaridou A, Andia ME, Saha P, Modarai B, Smith A, Botnar RM (2013) In vivo magnetization transfer and diffusion-weighted magnetic resonance imaging detects thrombus composition in a mouse model of deep vein thrombosis. Circ Cardiovasc Imaging 6:433-440

26. Papanikolaou N, Maniatis V, Pappas J, Roussakis A, Efthimiadou R, Andreou J (2002) Biexponential T2 relaxation time analysis of the brain: correlation with magnetization transfer ratio. Investig Radiol 37:363-367 